\title{
Contribuição dos sistemas de gestão de Carlos Matus para uma gestão comunicativa
}

\author{
A contribution from the Carlos Matus management \\ systems to communicative management
}

Águida Wi ch rowski Kopf 1

Virginia Al onso Hortale 2

\footnotetext{
1 Departamen to de Ciências da Saúde, Un iversidade Regional do Noroes te do Estado do Rio Grande do Sul/Unijui. Rua do Comércio 3.000, Bairro Un iversitário, 98700-000, Ijuí RS. aguida@unijui.tche.br. 2 Escola Nacional de Saúde Pública/Fiocruz.
}

Abstract The article discuss the contributions of management system of Carlos Matus to Health Organizations it has "as back drop" Comunicative Acting Theory by Jürgen Habermas for to apply in Health Organizations. This article proposes that proceedings of decision and operation of Health Organizations incorporate "matusianos Management Sys tems". It's necessary a bet ter integration and communication for a dialogal action in common agreement. The quality of participation depends on symmetrical reach for all agents in devel opment of abilities and reflections collectives which makes possible an intersubjective understanding.

Key words Matusianos management systems, Communicative management, Health organizations
Resumo O artigo discute as contribuições dos sistemas de gestão matusianos pa ra as organizações de saúde, tendo como pano de fundo a interpretação do referen cial do Agir Comunicativo de Habermas e sua conseqüente passagem para o plano das organizações de saúde. Defende que as instâncias de decisão e operação das organizações de saúde incorpo rem o referencial teórico-metodológico matusiano, e haja maior integração e comunicação intra e interunidades para a ação dialógica, o que demandará coordenação da ação acordada. O salto de qualidade depende de um alcance simétrico, para todos os agentes, no desenvolvimen to de habilidades pa ra a interação e reflexão coletiva, quepossibiliten o diálogo, exigência para um entendimento intersubjetivo.

Palavras-chave Sistemas de gestão, Gestão comunicativa, Organizações de saúde 
Introdução

A efetivação dos princípios e diretrizes do Sistema Único de Saúde (SUS) apresenta desafios fundamentais para o planejamento e a gestão dos serviços de saúde. A estes se impõem a necessidade de reestruturação, do tan do-os da flexibilidade e efetivid ade necessárias à de s centralização do setor e à complexidade e diversid ade de seus processos de trabalho, assim como mecanismos que possibilitem a participação efetiva de profissionais e usuários em seu direcionamen to e con trole. Isso exige uma coordenação da ação compatível com a natureza de sua missão e de seus processos de trabalho.

Uma nova gestão adequada às características particulares dos serviços públ i cos de saúde tem sido obj eto de preocupação de autores como Rivera (1991; 1995a; 1995b;1995c; 1995d), Rivera \& Artmann (1999); Dussault (1992); Junqueira \& Inojosa (1992); Cecílio (1993) e Campos (1997). Refletindo sobre os desafios mais urgentes para a gestão dessas organizações, esses autores enfatizam a necessidade de investir esforços em abord a gens gerenciais de scentralizadoras, que apostem em mecanismos que favoreçam a participação dos trabalhadores, o compartilhamento decisório e a formação de consensos, a cooperação interdisciplinar e a responsabilização cre s cente com os objetivos organizacionais.

A discussão aqui apresentada de senvolve-se no con texto da realizada por Rivera (1991; 1995a; 1995b; 1995) e Rivera \& Artmann (1999). Esses a utores têm enfatizado a necessidade de entender e informar a práxis na área de saúde na perspectiva de uma nova ética nas relações sociais, no plano das microrrelações que se desenvolvem no nível das organizações públicas de serviços de saúde, na gestão do cotidiano. Realiza uma discussão do planejamen to e da gestão em saúde na qual, fazendo um contrapon to às abord a gens norma tiva e estratégica $\mathrm{e}$, enfocan do a perspectiva da racionalidade comunicativa habermasiana, a ponta alternativas da sua passagem para o plano das organizações de saúde.

Este artigo é o desdobramento da tese de doutorado - "Ação Gerencial Dialógica - limites e possibilidades do Planeja men to Estratégico-Situ aci onal e da Gestão pela Qualidade", resultante da preocupação em investir em abordagens que possam conter elemen tos que se oponham ao predomínio da racionalidade instrumental nas organizações do mundo do tra- balho socialmente necessário, no caso nas organizações de saúde, e que ampliem espaços para a razão cen trada no en ten dim en to comunicativo, de base dialógica, en tre sujeitos capazes de falar e agir.

Tem como objeto de estudo os sistemas de gestão inscri tos na Teoria das Mac roorganizações de Carlos Matus, na perspectiva de suas possibilidades de contribuição como abordagem comunicativa. Enten demos que as reflexões desenvolvidas por esse autor têm uma $\mathrm{m}$ atriz instrumental, mas como bem já demonstrou Rivera (1991; 1995a), não obstante essa con dição, contêm el em en tos que podem integrar ou s er recon struídos para uma ação gerencial dialógica no campo das or ganizações de saúde.

Assim, discute-se neste arti go as con tribuições dos sistemas de gestão matusianos para as organizações de saúde, tendo como pano de fundo a interpretação do referencial do agir comunicativo e sua conseqüente passagem para o plano das or ganizações de saúde .

\section{A razão comunicativa de Jürgen Habermas como pano de fundo para o desenvolvimen to de novas formas de gestão do Sistema Único de Saúde}

Jürgen Habermas, que integra a segunda geração de pen s adores da Escola de Frankfurt, parte da discussão feita pelos teóri cos críti cos da geração anteri or, particularm en te Horkh eimer e Adorn o, para estrutu rar uma nova con cepção de racionalidade. Para esses teóricos, o saber produzido pelo Iluminismo, fundamentado na ciência positivista, ao invés de emancipar o homem, su bm eteu-o a um novo mito, repre s en $\mathrm{ta}-$ do pelo domínio da ciência e da técnica, substra to da razão instrumental.

Pa ra Habermas, uma vez que estaria excluída a identificação da emancipação do hom em com o progresso da ciência e da técnica, a via do esdarecimen to para o processo de form ação do sujeito e da sociedade fundamentar-se-ia numa nova racionalidade, a qual pre s supõe uma comunicação livre de coações e que se dá através da linguagem, meio universal da comunicação. Assim, define a sua con cepção de razão comunica tiva, situada historicamente na práxis social.

Procurando fazer mediação entre a racionalidade técnica e a racionalidade prática, $\mathrm{Ha}$ bermas constrói sua teoria social crítica com uma dimensão formal e pragmática que se de- 
senvo lve por meio da linguagem, e uma dimensão quase empírica, de uma teoria da socied ade ou do capitalismo avançado, que busca compreender os modos de incorporação da razão comunicativa nos movimen tos e instituições sociais que configuram a socied ade atual. Esta passaria por uma crise devi do ao avanço colonizador de subsistemas técnicos de ação racional com relação a fins.

Opõe a uma razão cen trada no suj ei to, que en con tra suas medidas críticas em critérios de verdade de conhecimento sobre objetos e coisas, uma razão cen trada na comunicação intersubjetiva. À ação rac i onal com relação a fins e a valores contrapõe o agir comunicativo, a poiado na herm en ê utica.

O con cei to de razão comu $\mathrm{n}$ i c a tiva propo s to por Habermas diz respeito à capacidade que tem uma fala argumentativa de unir sem coações e gerar con sen so, su peran do seus participantes a subjetivid ade inicial de seus pontos de vista pela via da argumentação discursiva de razões, o que permitiria unidade nas interpretações e uma definição intersubj etiva sobre um mundo objetivo e normativo que compartem, fren te aos mu n dos subj etivos de cada um (Habermas, 1987-I).

Em Habermas, para os processos de en tendimento, os três mundos conjuntamente (mundo obj etivo, mundo social e mundo subjetivo ) constituem o universo de referência dos seus participantes, já que, neste, cada indivíduo est abel eceria relações com o mundo obj etivo, com o mundo social e com o mun do subjetivo dos demais. Nestas relações com o mundo, estariam assentadas as racionalidades dos tipos de ação.

Unicamente a ação comunicativa tem na linguagem um meio para uma definição de situações que se refere simultaneamenteaos três mu n dos - objetivo, social e subj etivo. Na ação comunicativa, os atos de fala contêm um conteúdo proposicional, a oferta de uma relação interpessoal e expressam a intenção daquele que fala.

As ações sociais comunicativas correspondem a uma coordenação da ação em que os atores são movidos não por um cálculo egocêntrico de resultados, mas voltados para alcançar o en tendimento. Assim, nos processos orientados para o entendimento, pressupõe-se que estes sejam regulados pela observância de determinadas condições que permitam aos participantes ch ega rem a um acordo.

Os processos de en ten dim en to que têm por finalidade o consenso, de modo a distinguir-se do con senso ingênu o, têm de satisfazer nece ssariamente as condições de um assentimen to racional que se dá ao con teú do de um proferimen to, a uma pretensão de validade referida ao mundo objetivo, ao mundo social e ao mun do subjetivo, a poiada em algum tipo de razão ou argumento, reconhecido ou rejeit ado pelos outros participantes (Ha bermas, 1987-I).

$\mathrm{O}$ agir comunicativo tem como centro da discussão o mu n do da vida, como hori zon te no qual os agentes comunicativos se movem, o qual se vê re strin gidoe se modifica com as mudanças estruturais da sociedade (Habermas, 1987-I).

O mu n do da vida é integrado por três componentes estruturais: (a) cultura, que corresponde ao acervo de saber do qual os participantes da interação ex traem interpretações para se en ten derem sobre algo no mundo; (b) sociedade, en tendida como as ordenações legítimas através das quais os participantes da interação regulam suas pertenças a gru pos sociais, assegu ran do a solidariedade; e (c) pers onalidade, relacion ada às com petências que capacitam um indivíduo para a linguagem e para a ação, is toé, para tomar parte em processos de entendimen toe de afirmação da sua iden tid ade (Habermas, 1987-II).

$\mathrm{O}$ autor dem on s tra como o mu n do da vida se relaciona com os três con ceitos formais de mundo (mundo objetivo, social e subjetivo) que, na ação orientada para o entendimen to, formam a base das definições comuns na ação comunicativa.

Assim, a ação comunicativa, sob o aspecto funcional do en tendimento, serve à tradição e à ren ovação do saber cultural; s ob o aspecto da coordenação da ação, serve à integração social e à criação de solidariedade; e sob o aspecto da socialização serve à formação de identidades pessoais. Se ocorrem perturbações nos proce ssos de reprodução, essas se manifestam na cultura como perda de sentido; na socied ade, como anomia; e no âmbi to da personalidade, como psicopatologias. Cada uma das perturbações tem expressões nos demais componentes (Habermas, 1987-I).

A leitura da Teoria do Agir Comunicativo de Ha bermas obriga-nos a admitir que, ao enfocarmos abord a gens da teoria organizacional, nos situamos nos domínios da razão instrumental, portanto, submetidos às coações do mundo sistêmico. O que sugerimos é analisar, nos Sistemas de Gestão propostos por Matus, em que medida esses contêm em seus pressu- 
po s tos teóri cos e metodoló gi cos elementos que possam diminuir seus efeitos objetivos e instrumentais, possibilitandoampliar espaço e est a bel ecer certas condições para que, no âmbi to das organizações de saúde, possa a integração se fazer, não predominantemente pelos poderes econômico e administrativo, mas pela con strução de entendimentos lingüísticos entre os sujeitos organizacionais, crian do-se condições para um entendimen to intersubjetivo, racionalmente motivado e fundamentado em argumentações livres decoações.

No âmbi to das organizações de saúde, entendemos que não podemos reduzir seus trabalhadores às figuras do "hom em econômico", do "homem social", ou do "homem organizacional” (Chiavenatto, 1993), n em com preen der a gerência apenas como cadeia de transmissão das determinações gerais de qu em detém o poder (governante, proprietário, con selhos de direção, etc) (Campos, 1997), assim como os u suários a meros receptores das ações de saúde.

As teorias que têm orientado a administração pública compreendem um amplo espectro de visões que, no espaço con creto das organizações, confrontam racionalidades diversas de forma tensa e dialética (Rivera, 1995b). En tendemos que, no campo das or ganizações de saúde, essas distintas intencionalidades devem confluir para um processo dinâmico de construção de su jei tos coletivos (Merhy, 1995).

O SUS, ao constituir-se com base no pressuposto da saúde como um direito de cidadania, corre s pon de a um espaço que é fundamentalm en te públ i co. Aqui teríamos que con tra - a rgumentar, a partir da lógica habermasiana, que ainda assim nos situamos numa dimensão mediada pelo poder administrativo. Enten demos que a sustentabilidade e efetivação do SUS passam por uma relação de coerência en tre propósitos de govern o, métodos e organizações, como defendido por Testa em seu Postulado de Coerência (Rivera, 1989).

Adotar tal pressuposto implica utilizar a borda gens teórico-metodológicas que con tribuam para diminuir os efeitos objetivantes das matrizes instrum entais que, em geral, orientam o planejamento e a gestão das organizações de saúde.

\section{A racionalidade comunicativa e suas possibilidades de assimilação pela teoria organizacioml aplicada ao campo da saúde}

Às organizações de saúde, situadas na dimensão sistêmica, estaria interditado o inve sti m ento na racionalidade apontada por Habermas? Excluindo-se a possibilidade de intervenção imediata, a con tri buição para o aumen to da capacidade de auto-reflexão dessas organizações somente poderia emanar do meio circundante?

En ten demos que, se admiti do que os sujeitos or ganizac i onais se inserem e ex traem ori entações também do mundo da vida, a consideração do investimen to na ação comunicativa pode ser feita de s de que en tendida esta de forma processual, como espaços-mom en to a confrontarem-se, de forma dialética, com a racionalidade instrumental.

Nas organizações de saúde, investir em condições que possam contribuir para ampliar a a s cendência do mu ndo da vida sobre o sistema é o que pretendemos.

Para Rivera (1995a; 1995b), a busca da liqu efação dos limites en tre as esferas do mundo da vida e sistema, aplicados à gestão das organizações públicas de saúde, passaria pelo fortalecimen to de microprocessos que a integram na perspectiva de criar novas formas de relações entre os hom ens nas instituições. Pressuporia a necessidade de se trabalhar as instituições por dentro, no cotidiano, com pessoas con cretas, em processos mu i to len tos e apoiados numa diversidade de situações que possa configurar redes locais de estabelecimen to de solidariedade.

Os compon en tes do mu n do da vida dos atores seriam responsáveis, na organização, pela produção de significados possíveis de negociação, expressando mu n dos da vida diferenciados que cri a riam a possibilidade de conflito, enfrent ados por uma re s o lução discursiva ou por um a gir estratégica A difusão de val ores comuns geraria a possibilidade de con senso e a confirmação de regras formais e abstratas de um mundo da vida compartilhado, o que se confundiria com o con cei to de cultu ra institucional.

$\mathrm{O}$ autor salienta que o que agrega uma organização, da n do-lhe estabilidade, é o fenômeno da cultura. Partin do do con ceito de mun do da vida de Ha berm a s, e aplicando-o à realidace organizacional, o autor define o que corresponderia ao mundo da vida organizacional: o conjunto de saberes comu m en te partilh ado pe- 
la organização, a qui incluído o saber acerca do proj eto real sobre a missão; as formas de integração social que defin ema governabilidade e as capacidades de liderança e socialização dos su jei tos organizacionais, incluindo a responsabilização e a assunção de com promissos.

$\mathrm{O}$ agir comu n i c a tivo permiti ria que o mu ndo da vida dos atores organizacionais impregnasse a organização como totalidade sistêmica e fosse por ele regida, o que pressu poria substituir a categoria sujeito pela categoria intersubjetivid ade lingüisticam en te med $i$ ada.

Alguns autores que se têm dedicado à discussão da gestão no campo da saúde aportam contribuições importantes para a adequação das organizações, no sentido de que essas se tornem mais flexíveis e se adaptem ao movimento de descen tralização e à sua democratização, com a participação efetiva de profissionais e usuá rios na sua con du ção.

Dussault (1992) considera que as características das organizações de saúde geram formas particulares de gestão, e as entende como organizações profissionais. À gestão caberia conciliar quer a flexibilidade como coincidência da auton omia prof is s i onal com os obj etivo s organizacionais; qu er a descentralização, com a definição de mecanismos de con trole de qualidade e imputabilidade administrados de manei ra transparente. Já sua natu reza social pressuporia participação da comu nid ade na avaliação dos serviços.

Diante dessas características, o tipo mais adequ ado de gestão, p a raDussault, seria o consensual/colegiado, com ênfase em mecanismos de tom ada de decisão que envo lvam os profissionais sob a perspectiva da responsabilidade, do trabalho multi profissional e em integração com os usuários.

Também Junqueira \& Inojosa (1992) alertam que as caracterís ticas que assu m em a pre stação de serviços públ i cos de saúde, s eu proce sso de produção e o papel que o modelo assistencial passa a ter com o advento do SUS, requererem recontextualizar a gestão e envidar esforços em re sgatar a cidadania e a capacid ade criativa dos profissionais.

Para esses autores, inovação pede flexibilidade, des centralização e participação. Assim, $\mathrm{n}$ em a rigi dez prescritiva, $\mathrm{n}$ em a racionalidade estratégica, tecnicista ou tecnocrática seriam adequad as; a primeira, por castrar a criatividade profissional e penalizar o usuário; as últimas, por paut a rem-se pela re serva comunicativa, pela indução do com portamento e postura monológica, que favorecem o patrimonialismo, o corpora tivismo e o de s compromisso.

Também são propostos novos modelos organizacionais como o da or ganização atomizada e modular ou holográfica (Junqueira \& In ojosa, 1992), com unidades aut ô n omas a refl etir o todo or ganizacional.

Para Cam pos (1997), a administração tradicional das organizações públicas de saúde seria feita com a separação por categorias funcionais e estrutura de comando verticalizada. A gestão compatível com modelos assistenciais comprometi dos com a produção da saúde demandaria harmonizar a coordenação vertical com a idéia de coordenação horizontal. Assim, defen de um método para co - governar insti tu ições de saúde "produzindo liberdade e compromisso", por meio da criação de Unidades de Produção, as quais extinguirim os anti gos departamentos e seções recort adas segundo profissões, criando-se Unidades em con formidade com as lógicas específicas de cada processo de trabalho. Todos os participantes desses coletivos multiprofissionais comporiam um Colegiado para cada uma destas Unidades de Produção, os quais, por sua vez integrariam um Colegi ado Geral de Direção.

Cecílio (1993) também aponta a teoria do agir comunicativo de Habermas como essencial para en tender o interi or da instituição. $\mathrm{O}$ que se apres en t a ria como pano de fundo para a questão da gestão seria “a criação de um espaço público, consensualmente defin i doe compartilhado, no qual as diferen tes racion alid ades portadas pelos diferen tes grupos possam se cons en suar e se disputar".

Rivera (1995a), ao preocu par-se com a instrumentação geren cial, parte do pressupo s to de que a "gerência con temporânea" tem na comunicação seu eixo cen tral e que "gerir é comunicar, negociar, problematizar coletivamente e, essencialmente, produzir e acompanhar compromissos lingüisticamente mediado s".

Em síntese, nas organizações de saúde, a ampliação de espaços para a ação comunicativa dar-se-ia em três dimensões: (a) democratização das relações institucionais nos diferentes níveis hier á rquicos, permitindo a revisão crítica dos conteúdos políticos, administrativos e técnicos da organização necessários à tomada de decisão; (b) adoção de metodologias descentralizadas e participativas de processamento de probl emas e soluções, assegurando o adequ ado direcion a men to da missão; (c) o inve s timen to na maior re s ponsabilização de gestore s 
e trabalhadores de saúde sobre compromissos de ação, de forma a ga ra n tiro cumprim en toda missão.

Consideramos que processos que contemplemessas três dimensões permiti riam ampliar as condições para que se desvelem formas de ação estratégicas, e para que o mundo da vida dos sujeitos organizacionais possa, cada vez mais, informar processos de en ten dimen to que visem ao consenso, em vez de processos de coordenação da ação regidos exclu siva m en tepelo poder e o dinhei ro.

\section{Os sistemas de gestão con cebidos por Matus em sua teoria das macroorganizações}

$\mathrm{O}$ con ceito de mun do da vida, aplicado à gestão das or ganizações de saúde, em nosso en tendimento, corre s pon deria : - aos saberes prévios s obre saúde, de trabalhadores e usuários, na dimensão da cultura; - aos movimen tos sociais que se articulam para a solidari ed ade nas ações e decisões sobre saúde, na dimensão da integração social; - e às competências de trabalhadores e usu á rios para a manutenção de sua saúde e para tomar parte em instâncias deliberativas sobre saúde, na dimensão da socialização (Kopf, 1999).

Assim, respectivamente, acervo de saber, ordenações legítimas e personalidade podem in formar a or ganização das estrutu ras e os procedimentos organizacionais, dependen do do $\mathrm{m}$ a i or ou men or grau de abertu ra da gestão para o en tendimen to entre trabalhadores, confrontando-se aquelas com os critérios da dimensão sistêmica da sociedade, expressos nas organizações de saúde.

Já o con ceito de sistema, aplicado à gestão das or ganizações públicas de saúde, corresponde às restrições impostas pela intermediação dos poderes administrativo e econômico. Em nossa tradução, essas se ex pressam na estrutura, na hierarquia e nos processos decisórios adotados pelas organizações de saúde (Kopf, 1999). Nessa dimensão sistêmica, a linguagem serve apenas como meio indireto para uma coordenação da ação orientada por critérios de eficácia na ação sobre os fatos do mu n do obj etivo, e por critérios de êxito em um cálculo egoístico na busca de fins.

Para en ten der a gestão baseada nos fundamentos do Mun do da Vida dos atores, no que se refere ao nosso objeto de estudo, nos dete- mos em três sistemas de gestão matusianos, integrantes do denominado Triângulo de Ferro: Sistema de Agenda do Dirigente, Sistema de G erência por Operações e Sis tema de Petição e Prestação de Contas, os quais são informados por determinadas regras or ganizacionais.

Para Matus, a qualidade de uma macroorganização se dá em função de suas características fundamentais e de suas regras: de direcionalidade, de estrutura organizativa/departamentalização, de governabilidade e de responsabilidade (Rivera, 1995a).

As regras de direcion alid ade defin em a missão, os obj etivos e as funções de uma macroorganização e se expressam na Agenda do Dirigente, estando relacionadas ao uso do tempo e ao foco de atenção do diri gente.

Já as regras de departamentalização materializam as funções em dada estrutura organizacional, in tegrada por unidades de produção in termediárias e finais. Associadas às regras de governabilidade, definem como se dá o acesso aos recursos críti cos da organização pelos seus vários níveis hierárquicos, expressando-se no Sistema de Gerência por Operações, o qual com preen de a direção e ad min is tração por Objetivos, isto é, por módulos de ação que integram um plano, que se traduzem em operações, ações e subações, e que se articulam, por sua vez, com módulos orçamentários (programas, subprogramas, atividades), in tegrando uma atividade gerencial organizada em todos os níveis (Ma tus, 1993).

Segun do essa regra, as or ganizações seriam mais ou menos centralizadas, dependendo da possibilidade que cada nível hierá rquico tenha para definir os seus problemas de alto valor e do acesso aos recu ursos críti cos para enfrentá-los.

Para Matus, o poder concentrado reduz a capacidade de resposta oportuna do sistema diantedas mudanças da realidade e das oportunidades, enquanto o poder bem distribuído permite que cada nível organiza tivo se detenha em problemas de alto valor (Artmann, s/d).

Finalmente, as regras de responsabilidade são ex pressas pelo Sistema de Petição e Prestação de Contas, o qual tran s forma uma demanda neutra em informação sobre resultados de gestão (Artmann, s/d; Rivera, 1995a).

Sua eficácia dependeria de alguns requisitos: (a) constituir-se num sistema de prestações de contas público, em se tratando do nível político, ou que seja divul gado, no caso dos nívei s gerenciais, para que a população possa avaliar os seus resultados; (b) deve ser sistemático, com 
informação relevante e critérios de avaliação estabelecidos e conhecidos; (c) que a prestação de contas se refira a com promissos con c retos e precisos; (d) que seja verificável ou refutável, deven do por isso estar apoi ado num Si s tema de Informações; (e) que signifique um método de avaliação pessoal e institucional; e (f) que implique um sistema de sanções e recompensas (Matus, 1994b).

A nosso ver, a adoção dessas regras e respectivos sistemas, articulados entre si, permitem a cada nível organizacional trabalhar com problemas de alto valor, com direcionalidade, governabilidade e responsabilidade sobre probl emas e operações.

Enten demos que o foco em problemas de alto valor que o Si s tema de Agenda do Dirigente engendra, associado ao Si stema de Gerência por Operações, é on de reside o grande potencial para uma ação comunicativa mais efetiva nas or ganizações de saúde. Consideramos que o Sistema de Agenda do Dirigen te e o de Gerência por Operações con ferem dois requisitos fundamentais à descentralização do poder organizacional, em relação: à capacidade de cad a nível hierárquico se pronunciar sobre o que é relevante para a ação e o acesso aos recursos necessários; e à capacidade de integrar os trabalhadores para o en ten dim en to.

Complem enta o tratamento criativo e descentralizado de probl emas a proposta de Reengenharia Pública de Matus, na qual o autor define que as com petências devem ser descen tralizadas até um nível no qual todos decidam sobre problemas de alto valor para si (Matus, 1994b). Nessa proposta, o autor tece as suas considerações sobre os requisitos do processo de produção organizacional (Ma tu s, 1994a).

Ma tus defende que, quando o processo de produção não está sen do adequado, é necessário redefinir a organização a partir da responsabilização por produtos, en tre outros requisitos. (...) Quando una organización no funciona bien, es necessario: a) red efinir su macroo rganización, pa ra ajustarla a la delimitación clara de la responsabilidad por productos; $b$ ) inventar nuevos modos de acometer los rabajos y tareas requeridos pa ra producir los resultados terminales e intermedios, es decir, revolucionar los pro cesos microorganizativos y la rede de co nversaciones! ( Ma tus, 1994b).

O pon to cen tral dessa sua discussão é o direcionamento da missão a partir de uma definição muito clara dos produtos organizacionais e dos resultados a serem alcançados. (Ma- tus, 1994a). A crítica de Ma tus à divisão exa gerada do trabalho por especialidades fundamenta-se na tese de que a organização por unidades de produção é superior à departamentalização por áreas de conhecimento. Isso o leva a definir a seguinte orientação para a departamentalização: (...) Preci se la rede de producción de la institución, explicitando la tecnología de sus procesos. Identifique los principales productos terminales e interm edios que debe producir la organización y preci selos como cadenas de procesos (Matus, 1994b).

Enten demos que a departamentalização por Unidades de Produção, ao organizar-se pelo fazer, repres enta uma altern a tiva à organização tradicional dos processos de trabalho, nas organizações de saúde, com divisão de área de conhecimento. Essa configuração ajusta-se ao trabalho em equipe, rom pen do com as fron teiras disciplinares quando exigido, permitin do o pronunciamen to e a participação dos diversos atores envo lvi dos nos processos de produção.

Assim, complem en tem-se a departamentalização por Unid ades de Produção e o Sistema de Gerência por Operações, es tecorre s pon dendo a uma organização matricial por projetos, como expressão da divisão do trabalho em qu estões estratégicas paraaorganização, facult tadae exigida pelo seu referencial teórico-metodológico de planejam en to.

Visa também imprimir racionalidade à Agenda do Dirigente, permitindo que as Unidades de Produção lidem criativamen te com os problemas importantes da organização, a partir do tratamen to con sentân eo facultado pelo Sistema de Gerência por Operações.

Já no que se refere à responsabilização, o Sis tema de Petição e Prestação de Contas, ao definir a responsabilidade de cada unidade organizativa sobre os compromissos assumidos, juntamente com o Sis tema de Agenda do Dirigen te e com o de Gerência por Operações, destinam-se a definir o grau de ajustamento dos processos e resultados às necessidades definidas pela missão.

Considerado o principal do Triângulo de Ferro porqu e, segundoMatus, define se um sistema é de alta ou baixa responsabilidade, torn a efetiva a responsabilidade dos geren tes e de todo trabalhador sobre as operações, ações e subações que compõem um plano de ação, em todos os níveis hierárquicos.

Esse sistema trata fundamentalmente: - da relação entre propósitos, compromissos e resultados; - das causas das discrepâncias entre 
esses; - da eficiência, eficácia e oportunidade no en fren ta m en tode probl emas ena realização de operações; - da situação geral da gestão; dos valores e regras legitimados nos âmbitos social e organizacional.

Esse sistema gerencial para con ferir responsabilização, ainda que uma altern a tiva "aparelhística", ao estruturar-se em torno de operações/compromissos de ação, permite alocar responsabilidades para qu em exec uta e tem governabilidade sobre aquelas. Demonstra a interdependência entre esses elementos e os respectivos sistemas de gestão. Corre spon de a um pano de fundo, para o entendimento, como compromissos de ação legitimados organizacionalmente.

A nosso ver, trata-se de um sistema de gestão que articula re sponsabilização, direcionalidade e governabilidade, permitin do ainda informar um Si s tema de Avaliação de Desempenho.

\section{Considerações finais}

A análise dos sistemas de gestão matusianos, inscritos no chamado Triângulo de Ferro, aponta-nos alguns el em en tos que con si deramos relevantes para uma gestão comunicativa e responsabilizante.

O funcion a m en to po si tivo dos três sistemas de gestão apontados, em nosso entendimen to, gera um sistema de alta direcionalidade, governabilidade e responsabilidade, com coerência en tre si e em consonância com os requisitos de uma ação comunicativa, a qual consideramos n ecessária ao planejamen to e gestão das or ganizações de saúde.

O Sis tema de Agenda do Dirigente, cen trado em problemas de alto valor, corresponde a um planejam en to racional e compartilhado de processos e produtos, derivando em uma gerência descentralizada, o Sistema de Gerência por Operações.

Implicam, esses dois sistemas, grau signific a tivo de auton omia de cada nível hier á rqu ico, permitindo explicitar os valores dos trabalhadores envo lvi dos no processo de produção, ess enciais para articulação de autonomia, interdisciplinaridade, diferenciação e integração do s processos de trabalho, em suas re spectivas formações/profissões, com responsabilização em torno de com promissos de ação.

Entendemos que permitem maior espaço institucionalizado para considerar valores interdisciplinares, aportados das várias represen- tações do saber/fazer das organizações. Facultam o aporte: - do acervo de saber dos trabalhadores, de suas experiências e representações legítimas de socialização; - e das suas competências e habilidades pessoais para o enfrentamento das múltiplas dimensões envolvidas na a tenção às necessidades de saúde, seja no plano da percepção e acolhimen to das sentidas pelos usuários, seja no da organização das intervenções para respondê-las.

Em nossa experiência docente-assistencial constatamos uma carência efetiva de aplicação de referenciais teórico-metodológi cos de plan eja men to e gestão, no campo da saúde, no cotidiano do planejamen to de equipes locais de saúde, principalmente na realidade dos pequenos municípios brasileiros. Por isso, crescem em importância estu dos que vis emde s envo lver e aplicar referenciais teórico-metodológi cos de planejamen to e gestão em saúde, viáveis do pon to de vista técnico - organizacional, cognitivo e econômico.

Entendemos que os microprocessos políticos, administrativos e técnicos das organizações de saúde, que observamos em de senvolvimento nas múltiplas realidades de saúde dos municípios brasileiros, necessitam de referenciais factíveis que os ajudem a dar respostas efetivas às demandas cada vez mais complexas, de modo a respon derem aos princípios e diretrizes do SUS.

Isso exi ge desencadear movimen tos que articulema produção teórico-metodoló gica com sua dispon i bilização aos milhares de atores que estão implementando a descentralização da saúde no Brasil. Preferencialmente, referen ciais que re sga tem o exercício da cidadania e permitam uma gestão comu nic a tiva.

Demanda, também, o exame dos arranjos e instrumen tos normativos adotados, privilegiando os que trabalham com a possibilidade de uma coordenação da ação pela via da cooperação, leva n do em consideração as po s síveis diferenças de razões entre os proj etos dos distintos atores sociais implicados, sem prejuízo do alcanceda missão organizacional. Som en te assim, s erá possível con templar a diversid ade das realidades com as quais nos def rontamos num país como o Brasil, com dimensão continental.

E, para concluir, en ten demos que a organização dos processos de trabalho e de seus respectivos sistemas de gestão deve permitir, a nosso ver, que os diversos atores se pronunciem sobre o que consideram import a n te frente à realidade enfrentada, podendo esses se va- 
l erem de suas experiências e juízos de valor, obs ervan do os con tex tos em que atuam e, assim, subm eter suas diferen tes razões ao debate, ampliandoo espaço para um entendimento

Ressaltamos que o salto de qualidade depende de um alcance simétrico, para todos os agentes, no desenvolvimen to de habilidades para a interação e reflexão coletiva, que possibili tem o diálogo, exigência para um entendimen to intersu bj etivo.

A qualificação dos trabalhadores, voltada a uma participação não restrita aos aspectos téc- nicos, deve prover informações necessárias que lhes permitam usar seu raciocínio, de forma argumentativa, em todas as dimensões da organização, qualificando suas intervenções de modo que possam, autonomamente, participar das decisões. Estratégias de mobilização para uma participação não circunscrita à intervenção dos trabalhadbres aos aspectos técnicos da produção som en te são passíveis com a modificação con s entânea das estruturas de poder e a qualificação dos trabalhadores para a tomada de decisão em todos os aspectos da produção.

\section{Colaboradores}

AWK des envo lveu a con cepção te ó rica do estudo, a estrutura e os con teú dos do artigo.VAH auxiliou na sistem a tização dos con teú dos do artigo e na redação final.

\section{Referências bibliográficas}

Artmann E (s/d). Atualização da condensação traduzida do Planejamento Estratégico-Situacional-PES. Rel atório 2. Rio de Janeiro: ENSP/Fiocruz/CNPq.

Campos GWS 1997. Reforma da Reforma - repensando a saúde. (2a ed.). Hucitec, São Paulo.

Cecílio LC 1993. Inventando a mudança na administração pública - reconstituição e análise de três experiências na saúde. vols. 1 e 2 . Tese de doutoramento. Unicamp, Campinas.

Chiaven at to I 1993. Teoria geral da administração. vols. 1 e 2. Mc Graw-Hill, Makron Books, São Paulo.

Dussault G 1992. A gestão dos serviços públ i cos de saúde: características e exigências. Revista de Administração Pública 26(2):8-19.

Habermas J 1987. Teoria de la acción comunicativa. Racionalidad de la acción y racionalización social e crítica de la razón funcionalista. ( tomos I e II). Taurus, Madrid.

Junqueira LP \& Inojosa RM (1992). Gestão de serviços públicos de saúde: em busca de uma lógica de eficácia. Revista de Administração Pública 26(2):20-31.

Kopf AW 1999. Ação gerencial dialógica em saúde - limites e possibilidades do Planejamen toEs tratégico-Situacional e da Gestão Pela Qualidade. Tese de doutoramen to, ENSP/Fiocruz/Ministéri da Saúde, Rio de Jan eiro.

Matus C 1993. Pdítica, planeja men to \& governo. Tomos I e II. In stituto de Pesquisa Econômica Aplicada, Brasília.

Matus C 1994a. PES - Guia de analisis te o rico. Seminário de Gobierno y Planificación. Fundación ALTADIR, Caracas.

Matus C 1994b. El método PES: Reingenieria pública y la teoria de las co nversaciones: trabas y problemas. Fondo Editorial ALTADIR, Caracas (Colección Giencias y Técnicas de Gobi erno-2).
Merhy EE 1995. Planejamento como tecnologia de ge stão: tendências e deb a tes do planejamen to em saúde no Brasil, pp.117-148. In E Gallo. Razão e planejamen to-reflexões sob repolítica, estratégia e liberdade. Hucitec-Abrasco, São Paulo-Rio de Ja n ei ro.

Rivera FJU (org.) 1989. Planejamento e programação em saúde-umen foque estra tégico. Hucitec-Abrasco, São Paulo-Riode Janei ro.

Rivera FJU 1991. Jürgen Ha bermas: razão comunicativa e planejamento social - uma crítica ao enfoque estratégico. Tese de doutoramento. ENSP/Fundação Oswaldo Cruz. Rio de Ja neiro.

Rivera FJU 1995a. Comunicação e gestão organizacional por com promissos. Revista de Administração Pública 29(3):211-230.

Rivera FJU 1995b. Por um contraponto teórico entre o Planejamen to Estratégico-Situacional e o Controle de Qualidade Total (TQC) na saúde pública. Rio de Janeiro: In s tituto de Medicina Social/Uerj, no 110.

Rivera FJU 1995c. A gestão situacional (em saúde) e a organização comunicante. ENSP/Fundação Oswaldo Cruz, Rio de Ja neiro.

Rivera FJU 1995d. Agir comunicativo e planejamen to social (uma crítica ao en foque estratégico). Fiocruz, Rio de Jan ei ro

Rivera FJU \& Artmann E 1999. Plan ejamento e gestão em saúde: flexibilid ademetodo ló gica e agir comunicativo. Gestão do SUS: problemas, desafios e avanços. RevistaCiência e Saúde Col etiva 4(2):355-366.

Artigo apresentado em 18/10/2004

Aprovado em 16/06/2005

Versão final apresentada em 16/06/2005 